\section{Hydroxychloroquine retinopathy}

${ }^{1}$ The Oxford Eye Hospital, West Wing, John Radcliffe Hospital, Oxford, UK

\section{${ }^{2}$ Department of} Rheumatology, Nuffield Orthopaedic Centre, Oxford, UK

\section{Correspondence:} SM Downes, The Oxford Eye Hospital, West Wing, John Radcliffe Hospital, Oxford University Hospitals Trust Headley Way, Oxford OX3 9DU, UK

Tel: +44 (0)1865 234735;

Fax: +44 (0)1865 234515 .

E-mail: Susan.Downes@

ouh.nhs.uk

Received: 4 July 2016 Accepted in revised form: 24 August 2016 Published online: 10 March 2017

\begin{abstract}
Hydroxychloroquine (HCQ; Plaquenil) is used increasingly in the management of a variety of autoimmune disorders, with well established roles in dermatology and rheumatology and emerging roles in oncology. Hydroxychloroquine has demonstrated a survival benefit in patients with systemic lupus erythematosus; some clinicians advocate its use in all such patients. However, Hydroxychloroquine and chloroquine (CQ) have been associated with irreversible visual loss due to retinal toxicity. Hydroxychloroquine retinal toxicity is far more common than previously considered; an overall prevalence of $7.5 \%$ was identified in patients taking HCQ for greater than 5 years, rising to almost $20 \%$ after 20 years of treatment. This review aims to provide an update on $\mathrm{HCQ} / \mathrm{CQ}$ retinopathy. We summarise emerging treatment indications and evidence of efficacy in systemic disease, risk factors for retinopathy, prevalence among HCQ users, diagnostic tests, and management of HCQ retinopathy. We highlight emerging risk factors such as tamoxifen use, and new guidance on safe dosing, reversing the previous recommendation to use ideal body weight, rather than actual body weight. We summarise uncertainties and the recommendations made by existing HCQ screening programmes. Asian patients with HCQ retinopathy may demonstrate an extramacular or pericentral pattern of disease; visual field testing and retinal imaging should include a wider field for screening in this group. HCQ is generally safe and effective for the treatment of systemic disease but because of the risk of HCQ retinal toxicity, modern screening methods and ideal dosing should be implemented. Guidelines regarding optimal dosing and screening regarding HCQ need to be more widely disseminated. Eye (2017) 31, 828-845; doi:10.1038/eye.2016.298; published online 10 March 2017
\end{abstract}

\section{Introduction}

Hydroxychloroquine (HCQ: trade name, Plaquenil) and chloroquine (CQ: trade name, Aralen) are drugs established in the treatment of
IH Yusuf ${ }^{1}$, S Sharma ${ }^{1}$, R Luqmani ${ }^{2}$ and SM Downes' autoimmune disease and skin disorders, but are also emerging as a treatment option in oncology and paediatric inflammatory disorders. The expanding indications for HCQ, adjunctive use with biologic drugs and other disease modifying therapies, its role in the maintenance of disease remission, and favourable systemic safety profile during long-term use, predict a large and growing patient cohort on long-term HCQ therapy in developed nations. It is crucial therefore that Ophthalmologists are aware of the indications for $\mathrm{HCQ}$, identify risk factors for disease, request appropriate tests and know how to interpret them. The appropriate delivery of screening in accordance with a nationally agreed consensus recommendation is likely to minimize the risk of irreversible visual loss in this patient group.

\section{Indications for $H C Q$}

Chloroquine was developed in 1939, and through additional of an hydroxyl group, its analogue, HCQ was developed soon after and has been used since the 1960s. ${ }^{1}$ Chloroquine was originally developed and used to treat malaria. For this clinical indication, higher doses of chloroquine were used over a shorter period. Since then the use of chloroquine in the treatment of falciparum malaria has declined on account of widespread chloroquine resistance. HCQ was found to be less toxic and more effective than chloroquine. Hydroxychloroquine is now commonly used in a range of disorders (Table 1), most commonly non-organ specific autoimmune diseases such as systemic lupus erythematosus (SLE), rheumatoid arthritis, and mixed connective tissue disorders. ${ }^{2-4}$ HCQ may be used as a primary treatment for such disorders, or as an adjunctive therapy to reduce the required doses of other potentially more toxic drugs, such as biologic therapies.

HCQ modulates immune responses through several mechanisms. HCQ inhibits toll-like receptors 7 and 9 in dendritic cells, inhibiting interferon-alpha production. ${ }^{5} \mathrm{HCQ}$ has been shown to counteract the effect of extracellular oxidants from neutrophils, without reducing 
Table 1 Indications for Hydroxychloroquine treatment

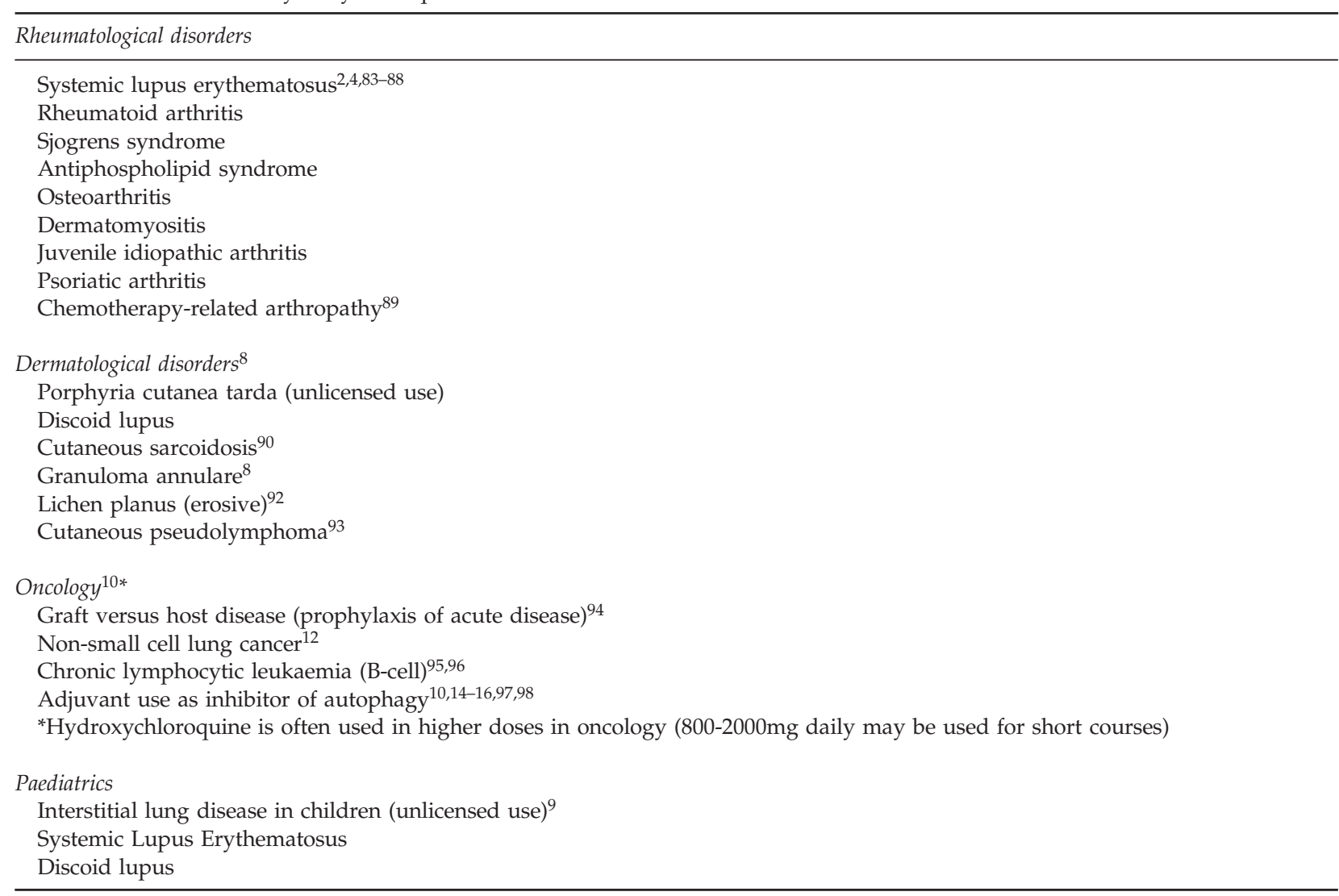

neutrophil cell counts, ${ }^{6}$ and disrupts the T-cell receptor dependent calcium signalling within $\mathrm{T}$ cells and thereby, antigen processing. ${ }^{7} \mathrm{HCQ}$ also preferentially induces apoptosis of a subset of effector $\mathrm{T}$ cells by inhibiting autophagy: a mechanism that is being explored through use of high dose HCQ in oncology trials. ${ }^{8} \mathrm{HCQ}$ increases the $\mathrm{pH}$ within intracellular vacuoles and interferes with lysosomal function, affecting the processing of antigenic peptides required to trigger autoimmune responses. ${ }^{9}$

The use of HCQ in patients with SLE is becoming more common. One study found that up to $50 \%$ of patients with SLE were prescribed HCQ, and this rate increased up to $90 \%$ in tertiary centres. ${ }^{10}$ Positive predictors of HCQ treatment in SLE were black race, haemodialysis, and being under the care of a rheumatologist; negative predictors were male gender and older age (over 45 years). ${ }^{11}$ It is likely that the use of HCQ in SLE will increase significantly following publication of the LUMINA study, which demonstrated a clear survival benefit of HCQ therapy in patients with SLE. ${ }^{2-4}$

The indications for HCQ use are expanding: it is increasingly used in photosensitive dermatological disorders such as discoid lupus and porphyria cutanea tarda. ${ }^{12,13}$ Experience with HCQ, and its favourable safety profile has encouraged its use in paediatric inflammatory disorders, such as juvenile idiopathic arthritis, discoid lupus, and SLE. ${ }^{12}$ It is used occasionally in paediatric interstitial lung disease as an unlicensed treatment. ${ }^{14}$

HCQ inhibits autophagy, a property that may reduce resistance to chemotherapeutic agents in oncology. ${ }^{15,16}$ At least five phase 1 clinical trials have been undertaken which combine high dose HCQ (800-2000 mg daily) in combination with erlotinib, ${ }^{17}$ vorinostat, ${ }^{18}$ temsirolimus, ${ }^{19}$ temozolamide, ${ }^{20}$ or bortezomib ${ }^{21}$ to determine whether autophagy inhibition is a useful strategy to overcome chemotherapeutic resistance. ${ }^{16}$

The use of HCQ is increasing due to: a growing list of clinical indications, increasing confidence amongst physicians to start treatment, few systemic adverse effects necessitating treatment cessation and adjunctive use in conjunction with a range of primary therapies (such as biologic agents). The LUMINA study is likely to significantly increase the number of individuals with SLE taking long-term HCQ. After 5 years, these patients will require screening. ${ }^{2,22-25}$ Regardless of the threshold at which screening is advocated, the number of patients 
referred for ophthalmological evaluation will inevitably increase, as will the numbers of patients developing HCQ retinopathy.

\section{Contraindications to $H C Q$}

There are relatively few contraindications to HCQ. The manufacturer lists them as follows: known hypersensitivity to 4-aminoquinolone compounds, pre-existing maculopathy, and pregnancy. ${ }^{12}$ The use of HCQ in pregnancy is controversial; although HCQ is known to cross the placenta, it has been associated with improved outcomes for mother and child in the context of maternal SLE. ${ }^{26}$ A cohort study demonstrated reduced neonatal morbidity in mothers with SLE by reducing the rate of prematurity and intrauterine growth restriction. ${ }^{27}$ This has led to the recommendation by some authors that HCQ be continued during pregnancy and lactation, although it is unlicensed in this context. ${ }^{5}$

\section{Dosing}

HCQ is available in 200 or $400 \mathrm{mg}$ film-coated tablets. The manufacturer, ${ }^{12}$ and previous best practice clinical guidelines, ${ }^{22,24}$ have traditionally suggested dosing based on ideal body weight (IBW), rather than actual body weight $(\mathrm{ABW})$, to reduce the risk of retinal toxicity. The maximum dose of HCQ of $<6.5 \mathrm{mg} / \mathrm{kg}$ of IBW per day (mg/kg of IBW/day) has traditionally been considered as safe for most adults. However, recent evidence suggests that dosing based on ABW is more predictive of toxicity and is more accurate over a broad range of body habitus. ${ }^{25,28}$ Melles and co-authors ${ }^{28}$ suggested that $5.0 \mathrm{mg} / \mathrm{kg}$ of ABW/day corresponded approximately to a dose of $6.5 \mathrm{mg} / \mathrm{kg}$ of IBW/day because of the average body habitus of their study population. This change in prescribing recommendation for HCQ may be more practical in rheumatology clinics, as it has been shown that calculating dose of HCQ based on IBW is not undertaken on the majority of HCQ patients (Worth et al, 2016 - unpublished data). Very thin patients are at risk of receiving a higher than the recommended dose of HCQ if IBW is used (at dose of $6.5 \mathrm{mg} / \mathrm{kg}$ of IBW/day), and obese individuals or those of short stature, in particular, are at risk of retinal toxicity if $\mathrm{ABW}$ is used (at dose of $6.5 \mathrm{mg} / \mathrm{kg}$ of $\mathrm{ABW} /$ day). ${ }^{24}$ The subsequent dose will either be 200 or $400 \mathrm{mg}$ per day. Paediatric patients with an IBW of $<31 \mathrm{~kg}$ cannot take HCQ as the $200 \mathrm{mg}$ tablet will exceed $6.5 \mathrm{mg} / \mathrm{kg}$ of IBW/day, and a safe dose cannot be prescribed. ${ }^{12}$ The 2016 AAO guidelines do not suggest changing the dose of HCQ for children, and this should continue to be calculated based on IBW. ${ }^{25}$

Serum levels of HCQ can be measured in patients with known hepatic or renal failure, where the levels of HCQ are more difficult to predict based on ABW or IBW calculations alone. The drug dose can then be adjusted accordingly. ${ }^{12}$

\section{Systemic toxicity}

The manufacturer lists reported adverse events attributed to HCQ therapy. ${ }^{12}$ They suggest caution in patients with known hepatic or renal dysfunction, or drugs known to compromise function of these organs. HCQ has been reported to cause severe hypoglycaemia in patients with diabetes taking hypoglycaemic drugs, and rarely, bone marrow suppression or skeletal muscle weakness ${ }^{12}$ There have been reports of fulminant hepatic failure in patients with no pre-existing liver disease soon after the initiation of HCQ treatment. ${ }^{29,30}$ It is considered that $<1 \%$ of patients taking conventional doses of HCQ suffer elevation of liver enzymes on serum evaluation, and taken together, these reported cases are considered to represent idiosyncratic drug reactions. ${ }^{29}$

\section{Efficacy of HCQ in systemic disease}

HCQ has been demonstrated to reduce the risk of diabetes, thrombosis and dyslipidaemia in patients with $\mathrm{SLE}^{5}$, and demonstrates a protective effect on renal function in lupus nephritis. ${ }^{3,5}$ A landmark clinical study in the United States (LUMINA) identified a survival benefit with HCQ in patients with SLE, perhaps due to a combination of these effects. ${ }^{2}$ Some clinicians now advocate its use in all patients with SLE. ${ }^{5}$ Its use has been demonstrated to reduce the risk of thrombosis in patients with anti-phospholipid antibodies. ${ }^{31}$ The clinical evidence for HCQ therapy across all listed indications is referenced in Table 1.

\section{Duration of therapy}

The duration of HCQ therapy for most rheumatic diseases is not clearly defined. The general principles of managing rheumatoid arthritis are to achieve disease control as rapidly as possible, usually with combinations of different disease modifying agents, often including HCQ.

Subsequently, once patients achieve remission, the aim is to minimise drug doses where feasible, but this is usually applied to reducing doses of the more potent and potentially more toxic anti-rheumatic drug therapy, rather than changing the dose of HCQ. In a recent study, the use of long-term triple therapy of HCQ, sulfasalazine and methotrexate was compared with use of a biologic agent in combination with methotrexate and showed the advantages of using a biologic drug in combination; no significant toxicity from the use of HCQ in 1160 patients was reported. ${ }^{32}$ A large Cochrane review supports the 
role of triple disease modifying anti-rheumatic therapy (including HCQ) in the management of rheumatoid arthritis. ${ }^{33}$ A large Finnish study of combination therapy including HCQ has reported effective control of rheumatoid arthritis without any significant increase in drug toxicity over an 11-year period comparing monotherapy with combination therapy. ${ }^{34}$ The implication from these studies and in clinical practice is that rheumatologists favour long-term use of combination therapy for disease control in rheumatoid arthritis.

\section{HCQ retinopathy}

\section{Mechanism of retinal toxicity}

The mechanism of HCQ related retinal toxicity is uncertain. One recent study identified that both chloroquine and HCQ strongly inhibit the uptake activity of an organic anion transporting polypeptide 1A2 (OATP1A2), expressed in human retinal pigment epithelium (RPE) cells, which is involved in the recycling of all-trans-retinol, suggesting a possible effect of HCQ on the visual cycle. HCQ markedly inhibited the uptake of all-trans-retinol in human embryonic kidney cells (HEK293) and primary human RPE cells. ${ }^{35}$ Increased permeability of the RPE in response to HCQ was also demonstrated in vitro. ${ }^{36}$

An evaluation of ocular tissues after long term administration of chloroquine in rhesus monkeys revealed widespread binding of chloroquine in pigmented ocular tissues: the RPE, iris, choroid and ciliary body with eventual accumulation observed in the retina. ${ }^{37}$ Drug accumulation within the RPE may explain the progression of HCQ retinopathy after drug cessation in some patients. This may result in outer retinal and photoreceptor degeneration with later, secondary degeneration of the RPE. It is currently unclear why the photoreceptors in the parafovea/ perifovea are most vulnerable to the toxic effects of HCQ seen clinically.

An alternative explanation is that the primary site of toxicity in HCQ/CQ retinopathy is the photoreceptor layer with secondary degeneration of the RPE. ${ }^{38}$ Spectral domain optical coherence tomography (OCT) data suggest degeneration in HCQ retinopathy is localised to the outer retina/photoreceptor layer, ${ }^{39}$ before any structural RPE damage is apparent. This is a finding seen in experimental studies of HCQ/CQ retinopathy. ${ }^{37}$ HCQ is known to affect the $\mathrm{pH}$ and function of lysosomes, and it may be that phagocytosis of outer segments of photoreceptors, lysosomal function with the RPE and autophagy are impaired within the RPE, affecting photoreceptor cell membrane stability and, ultimately, function. Further work is required to establish the exact sequence of events in HCQ retinal toxicity.

\section{Risk factors}

The risk factors detailed below are recognised to increase the risk of HCQ retinopathy. The sum of risk factors can provide an estimation of risk for a given individual. ${ }^{40}$ Some risk factors are preventable: the most important of these is appropriate drug dosing of $\mathrm{HCQ}$, especially CQ. ${ }^{40}$

The American Academy of Ophthalmology (AAO) in their 2016 recommendations defined 'major risk factors' on the basis of new data from a landmark epidemiological study of 2361 patients who had used HCQ continuously for greater than 5 years. ${ }^{28}$ The overall prevalence of HCQ retinopathy in this study was $7.5 \%$, but increased to around $20 \%$ after 20 years of therapy. ${ }^{28}$ 'Major risk factors' listed by AAO (2016) include HCQ dose $>5.0 \mathrm{mg} / \mathrm{kg} \mathrm{ABW}$ or CQ dose $>2.3 \mathrm{mg} / \mathrm{kg} \mathrm{ABW}$, duration of use $>5$ years (assuming no other risk factors), renal impairment (defined as reduced glomerular filtration rate), tamoxifen use, and macular disease. ${ }^{25}$

\section{Duration of HCQ use}

The Royal College of Ophthalmologists (RCOphth) joint guidelines from 2009 suggest referral to an Ophthalmologist for patients who have received continuous HCQ therapy for more than 5 years. ${ }^{22}$ It is made clear in the AAO guidelines (2011 and 2016), that the screening schedule should begin after 5 years of HCQ/CQ therapy, and that it is a 'major risk factor' that increases the risk of retinal toxicity. ${ }^{24,25}$ The AAO guidelines also state that screening may begin before 5 years of therapy if additional risk factors are present (renal failure, tamoxifen use, etc). The manufacturers of HCQ do not specify a duration of drug use after which ophthalmological evaluation should be sought. ${ }^{12}$ Table 2 details the differences in screening recommendations and risk factors for HCQ/CQ retinopathy.

\section{Daily dose of HCQ}

HCQ has been traditionally prescribed at doses below $6.5 \mathrm{mg} / \mathrm{kg}$ of IBW/day (below $3.0 \mathrm{mg} / \mathrm{kg}$ IBW/day for $\mathrm{CQ})$, calculated from IBW using the patient's height and one of a number of specific algorithms. ${ }^{41}$ Dosing by IBW may be a source of uncertainty amongst clinicians, and has led to many patients receiving a dose in excess of the correctly optimised value. ${ }^{42}$ In a retrospective review of 675 patients on HCQ, Walvick and co-authors identified that $56 \%$ of females and $46 \%$ of males were receiving higher than the recommended dose of HCQ. ${ }^{42}$ This may be related to the fact that $88.5 \%$ of patients using HCQ had a body weight in excess of their IBW in the largest series of 3995 patients, and $53.6 \%$ of patients were taking a HCQ dose greater than $6.5 \mathrm{mg} / \mathrm{kg} \mathrm{IBW} /$ day. ${ }^{43}$ 


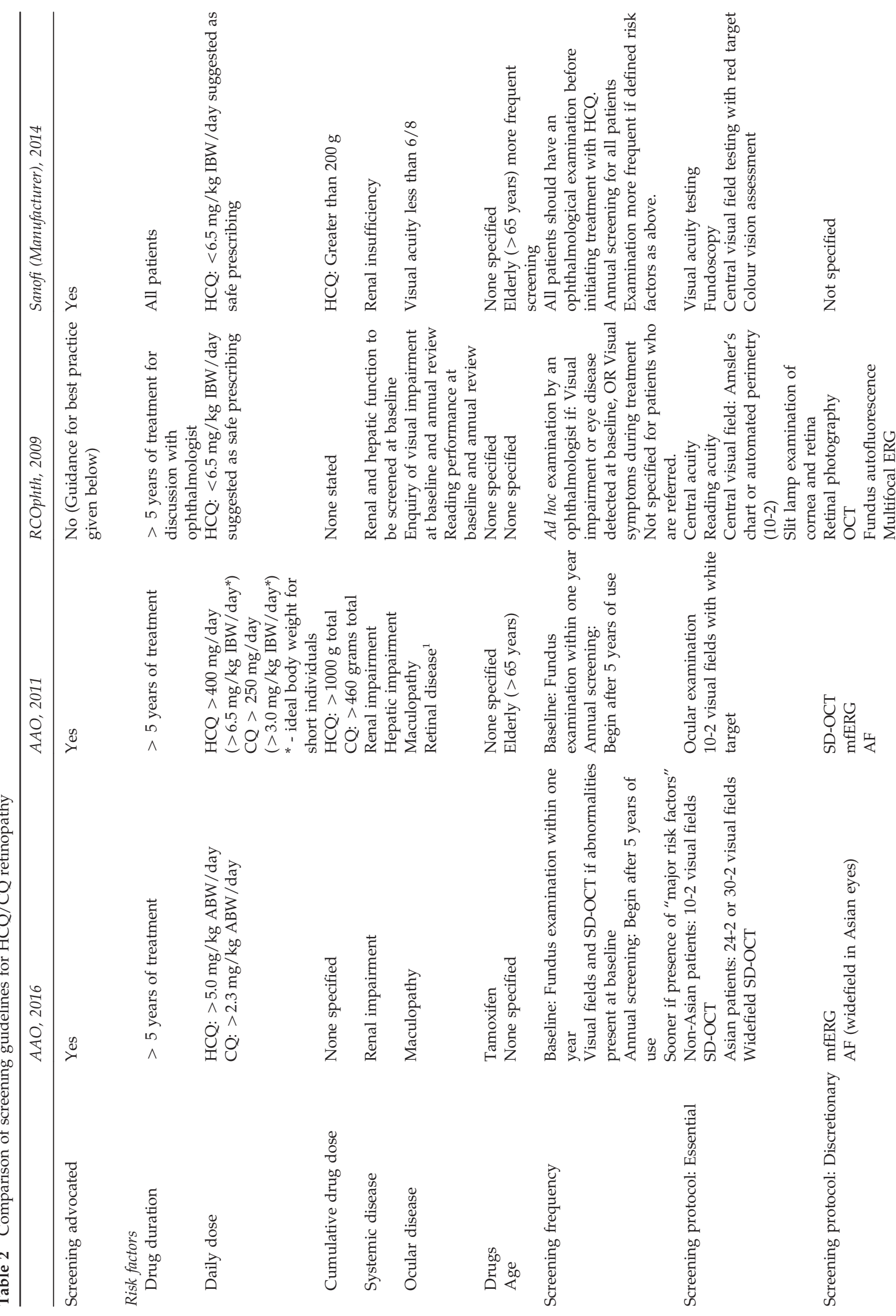


In an audit performed locally of HCQ dosing, we identified that $10 \%$ of patients were taking a dose of HCQ in excess of the recommended dose based on $\mathrm{ABW}$, and $30 \%$ were being overdosed based on IBW (unpublished data).

However it is now accepted that ABW is the best method rather than IBW and this is largely based on the influential epidemiological study of HCQ retinopathy undertaken by Melles and Marmor, who determined that ABW is a better predictor of retinal toxicity than IBW. ${ }^{28}$ Since patients were typically $25-30 \%$ heavier than their IBW; $6.5 \mathrm{mg} / \mathrm{kg}$ of IBW/day translates to $\sim 5.0 \mathrm{mg} / \mathrm{kg}$ $\mathrm{ABW} /$ day, which the authors defined as the threshold between judicious and excessive HCQ use. The sensitivity and specificity of ABW was greater than IBW in predicting toxicity, and the size of the study permitted risk stratification based on dose of HCQ/ $\mathrm{kg} \mathrm{ABW/day:}$ the risk of HCQ retinopathy is 2-3 times higher with doses of 5.0 compared with $4.0 \mathrm{mg} / \mathrm{kg} \mathrm{ABW} /$ day. Dosing based on ABW rather than IBW has now become the recommended means of calculating the safe dose of HCQ as per the recent AAO guidelines 2016. ${ }^{25}$

Ophthalmologists and rheumatologists should be aware of this important change in best practice for drug dosing. Dosing by ABW is more practical and eliminates the need to measure patient height and select an appropriate formula in order to calculate the IBW. It is likely that dosing by ABW will gain popularity amongst screening guideline variations worldwide, given this data, although care must still be taken when prescribing HCQ/ CQ for patients at extremes of height and weight: short or obese individuals are at most risk of being prescribed a higher than recommended dose. ${ }^{24,41,42}$

\section{Cumulative dose of $\mathrm{HCQ}$}

Cumulative dose has traditionally been used in research studies as a risk factor for HCQ/CQ retinopathy, and is therefore considered separately here. The 2011 AAO guidelines stated that a cumulative dose of $>1000 \mathrm{~g}$ increases the risk of retinopathy (equating to 6.85 years of treatment at $400 \mathrm{mg}$, and 13.7 years of treatment at $200 \mathrm{mg}) .{ }^{24}$ However, the conclusions drawn on the significance of the cumulative dose are inconsistent. It was omitted entirely from the pivotal epidemiological study by Melles and Marmor, and was consequently omitted as a major risk factor in the recent 2016 AAO guidelines, which are based heavily on this study. ${ }^{25}$ It may be argued that defining greater than 1,000 grams of cumulative HCQ dose as high risk does not add to these guidelines, as a patient taking greater than 1,000 grams of HCQ will have been treated for longer than 5 years in any case unless the traditionally accepted maximum daily dose of HCQ ( $<6.5 \mathrm{mg} / \mathrm{kg}$ of IBW/day) is exceeded. 


\section{Ocular}

The manufacturers of HCQ suggest that pre-existing maculopathy is a contraindication to treatment, ${ }^{12}$ and the AAO criteria state it as a risk factor for the development of HCQ retinopathy. ${ }^{24,25}$ There are no specific data to demonstrate that patients with pre-existing macular disease are more susceptible to HCQ retinopathy, although subtle parafoveal structural and functional abnormalities secondary to HCQ may be difficult or impossible to detect with SD-OCT, AF or mfERG in the context of pre-existing macular disease. Macular disorders should be identified at baseline by Ophthalmologists as part of AAO (2011) guidelines ${ }^{24}$ and baseline examination within the first year of treatment is advised in the 2016 guidelines. ${ }^{25}$ This will only be identified, according to RCOphth joint guidelines (2009) if the patient has visual symptoms at baseline and is then encouraged to see an Optometrist to determine whether the macula is affected, with an onward referral to an ophthalmologist if so. ${ }^{22}$ The authors of RCOphth joint guidelines advocate Amsler's screening from Rheumatology clinics by the prescribing physician. ${ }^{22}$ Interestingly this has been omitted from the more recently published AAO guidelines (2011 and 2016). ${ }^{24,25}$ Isolated drusen with good photoreceptor function should not be considered a contraindication to HCQ treatment: a baseline SD-OCT, and AF imaging with a visual field test should be carried out in this context. ${ }^{25}$

\section{Systemic}

\section{Age}

Age was excluded as an independent 'major risk factor' in the AAO revised guidelines, 2016, ${ }^{25}$ although it was considered a major risk factor the 2011 AAO guidelines. ${ }^{40}$ Several reports have documented HCQ retinopathy occurring in elderly individuals despite a dosing regimen within the therapeutic guidelines. ${ }^{44}$ One study of 32 individuals taking HCQ found that electroretinography was able to detect changes over the course of treatment in elderly patients (over 65 years), not present in younger patients. ${ }^{45}$ Maculopathy, and deteriorating renal function may be associated with increasing age; both considered major risk factors in isolation.

\section{Renal failure}

HCQ is renally excreted and consequently renal impairment is likely to increase the circulating concentration of the drug and risk of toxicity. In a large study evaluating the systemic factors that determine serum concentration of HCQ, renal failure was associated with a significantly higher serum HCQ concentration. ${ }^{46}$ Melles and Marmor were the first to quantify the risk of
HCQ retinopathy by the degree of renal failure (using glomerular filtration rate: GFR): a 50\% decline in the GFR was associated with a doubling of the rate of HCQ retinopathy (odds ratio (OR): 2.08). ${ }^{28}$ SLE may result in lupus nephritis in some patients, and end-stage renal failure. However, HCQ has a protective effect on lupus nephritis in this patient group. ${ }^{3}$ Interestingly, although HCQ is partially metabolised by the liver, liver disease was not associated with increased risk of HCQ retinopathy. ${ }^{28}$

\section{Tamoxifen}

Tamoxifen has come under scrutiny as potentially causing a dose-dependent maculopathy that may concurrently increase the risk of HCQ toxicity. A retrospective casecontrol series of 2361 patients taking HCQ for at least 5 years found that there was a significant increased risk of retinopathy in patients taking tamoxifen (OR: 4.59). It is notable that tamoxifen use was found to have a greater odds ratio than renal impairment (OR: 2.08), duration of use (OR: 3.22) but not daily dose (OR: 5.67). ${ }^{28} \mathrm{HCQ}$ retinopathy in this patient group correlated with cumulative tamoxifen dose. ${ }^{28}$ Tamoxifen use is mentioned specifically as a 'major risk factor' for HCQ retinopathy in the recent revised AAO guidelines, 2016. ${ }^{25}$ Tamoxifen is often used for up to 10 years or more following the diagnosis of oestrogen receptor positive breast cancer, and licensed for the prevention of breast cancer in women considered high risk.

Crystalline retinopathy, macular oedema, pigmentary retinopathy, and reversible corneal changes have been described in patients taking tamoxifen. ${ }^{47}$ The mechanism of retinal toxicity of tamoxifen is uncertain. One study evaluated the effect of tamoxifen on cultured RPE cells, finding lysosomal destabilization and cathepsin release prior to regulated cell death in cultured RPE cells. ${ }^{48}$ A further study identified a profound decrease in the activity of lysosomal enzymes in cultured RPE cells with both tamoxifen and CQ. ${ }^{49}$ These drugs in combination may act synergistically therefore to further adversely affect the phagocytosis of the retinal outer segments as a common disease mechanism.

\section{High dose HCQ in oncology trials}

HCQ is often used at higher doses than is typical in inflammatory disease in oncology trials (800-2000 mg daily), ${ }^{16}$ albeit in short courses with an intermittent dosing schedule. Rapid onset toxic retinopathy has been reported in two out of seven patients being treated with erlotinib and high dose HCQ (1000 mg daily) for nonsmall cell lung cancer. ${ }^{50}$ The affected patients developed HCQ retinopathy at 11 and 17 months of exposure and 
were detected by screening in the pre-symptomatic stage of disease. ${ }^{50}$

In a series of 12 patients who underwent HCQ therapy (800 mg/day) for chronic Graft versus Host disease, three developed retinal toxicity with scotomata on Amsler's grid, central visual field testing (10-2) and mfERG. Median duration of treatment in this series was 22.8 months, and median adjusted daily dose was $11.5 \mathrm{mg} / \mathrm{kg} /$ day. This small series suggests a potential increased incidence of HCQ retinopathy in the context of pre-existing graft-versus-host disease and earlier development of retinal toxicity. ${ }^{51}$ Graft-versus-host disease has itself been associated with a microvascular retinopathy, and this observation of suggested HCQ toxicity in this series should be interpreted in this context. $^{52}$

\section{Pharmacogenomics}

As a bull's eye maculopathy phenotype is also described in inherited retinal degeneration, it could be possible that genetic variants in gene(s) involved in retinal function or structure could be contributing to the phenotype rather than HCQ, or HCQ might be potentiating the effects of an underlying genetically determined disorder. This could explain the apparent idiosyncratic retinal reaction of patients presenting with retinal toxicity. The bull's eye maculopathy phenotype has been described in association with ABCA4 pathogenic variants. Shroyer et $a l^{53}$ carried out mutational analysis of the ABCA4 gene in 8 patients taking HCQ. Five patients were found to have variants in this gene. Interestingly a different study reported a protective effect in association with 3 common $A B C A 4$ variants and reported that they appeared to reduce susceptibility to HCQ/CQ maculopathy. ${ }^{54}$ This finding, in combination with the discovery of $A B C A 4$ gene mutations in the majority of patients with HCQ/CQ retinopathy in a single series could mean variants in this gene may have a modifier role. ${ }^{53}$ However ABCA4 polymorphisms/variants are common $^{55}$ and their relevance to HCQ toxicity is not yet proven. A large study would be required to investigate this further.

Of course, if there are unusual features present, such as the presence of flecks as seen in some ABCA4 retinopathies, ${ }^{56}$ or any other features suggestive of an inherited retinal dystrophy genetic analysis should be considered. ${ }^{53,57}$ In the future, pharmacogenomics may well play an important role in increasing the safety of HCQ treatment by excluding those genetically predisposed to macular toxicity.

\section{Epidemiology}

Studies evaluating the incidence of HCQ/CQ retinopathy are typically retrospective, cohort studies of populations of patients taking HCQ in a single centre, or registry data from multiple centres. ${ }^{58}$ Cross comparison of the incidence of HCQ retinopathy in different study populations is problematic; definitions of HCQ retinopathy and techniques used to identify retinopathy vary and susceptibility to HCQ retinopathy may also differ between study populations.

Older studies are thought to have underestimated the prevalence of HCQ retinopathy as cases were usually detected when apparent on fundus examination and included those on short-term HCQ/CQ treatment who are at low risk of disease. New diagnostic tools and improved recognition of abnormal results has permitted more sensitive detection of retinopathy before fundus abnormalities become manifest. ${ }^{28}$

Melles and Marmor published the most influential epidemiological study of HCQ retinopathy in $2014^{28}$ and the AAO produced updated guidance on HCQ screening as a result of this study in $2016 .{ }^{25}$ Their case-control study identified a study population of 3.4 million individuals, with 2361 patients who had taken HCQ for more than 5 years, and were evaluated by SD-OCT and visual field testing. A large study population permitted detailed analysis of risk factors with sub-group analysis (such as risk of retinopathy in different ranges of doses by weight). The overall prevalence of HCQ retinopathy was $7.5 \%$, but this increased to around $20 \%$ after 20 years of use for those taking 4.0-5.0 mg/ kg ABW/day and can exceed $50 \%$ at 20 years for those taking greater than $5.0 \mathrm{mg} / \mathrm{kg}$ $\mathrm{ABW} /$ day. Odds ratios were attributed to risk factors: daily dose (OR: 5.67), duration of use (OR: 3.22), kidney disease (OR: 2.06) and tamoxifen use (OR: 4.59). These data have led to an evidence-based update of the AAO HCQ guidelines, and thus promoted the safe prescribing and screening of HCQ retinopathy by allowing a risk calculation for each patient according to daily dose, duration of therapy and other major risk factors. ${ }^{28}$ These estimates are helpful for Rheumatologists, Ophthalmologists and patients. Providing an evidencebased argument for dosing according to ABW rather than IBW is one of the key outcomes of this study adopted by the AAO guidelines. ${ }^{28}$

\section{Clinical features}

HCQ and chloroquine retinopathies, once symptomatic, are characterised by abnormalities of the retinal pigment epithelium, which are detectable clinically, and may later develop into the classic appearance of 'bull's eye maculopathy' with central potentially extensive, 
concentric, parafoveal retinal pigment epithelial loss. This clinical state is associated with severe and irreversible visual loss that may be complicated by secondary cystoid macular oedema, epiretinal membrane and other sequalae. ${ }^{59}$

Screening for HCQ/CQ retinopathy may detect structural or functional changes in the macula prior to the development of symptoms or fundus abnormalities. Although drug cessation, partly due to long half-life of the drug, does not always arrest progression of toxicity, it can limit the extent of structural and functional deficits and reduce the risk of irreversible visual loss. ${ }^{60}$ One study identified improvement of visual function in HCQ retinopathy following drug cessation, but no improvement in structural parameters. ${ }^{61}$

A key recent finding was the identification of variable distributions of disease in HCQ/CQ retinopathy on the basis of race. ${ }^{62}$ Patients of European descent exhibit a classic parafoveal pattern (2-6 degrees from the fovea) of structural and functional deficits, were more likely to manifest an extramacular or pericentral (more than 8 degrees from the fovea) pattern of disease in the early stages with structural and functional abnormalities identified near the vascular arcades. ${ }^{62}$ Pericentral HCQ retinopathy was seen in $50 \%$ of Asian patients in this series, but only $2 \%$ of Caucasian patients. ${ }^{62}$ Black and Hispanic patients demonstrated a predominantly parafoveal distribution of disease, although the pericentral distribution or mixed distribution of disease in these two racial groups was seen slightly more often than in Caucasian subjects. ${ }^{62}$ The reason for the different disease phenotype in these different ethnic groups is unclear, but not thought to be related to ocular pigmentation alone. ${ }^{62}$ This distinction is clinically noteworthy: Asian patients undergoing screening for HCQ retinopathy will require wider field structural (wide-field or off-axis SD-OCT and AF imaging) and functional investigation (24-2 or 30-2 visual field rather than 10-2) than Caucasian patients (Figure 1).

HCQ is associated with the development of vortex keratopathy or corneal verticillata, which can rarely result in visual disturbance. This change is entirely reversible on drug cessation, and is reported to be more common with CQ than HCQ. It bears no direct relevance to the severity of retinal disease.

\section{Screening and monitoring in $\mathrm{HCQ} / \mathrm{CQ}$ patients \\ Baseline screening}

Baseline screening is suggested by the AAO guidelines of 2011 and 2016 within one year of starting HCQ/CQ, and by the manufacturers before starting HCQ in order to exclude pre-existing maculopathies, with visual fields and SD-OCT recommended at baseline in the 2016 criteria if any abnormalities are found. RCOphth do not recommend baseline screening, unless there are visual symptoms when HCQ/CQ is started, or if the patient has been referred by an Optometrist. There is no specific

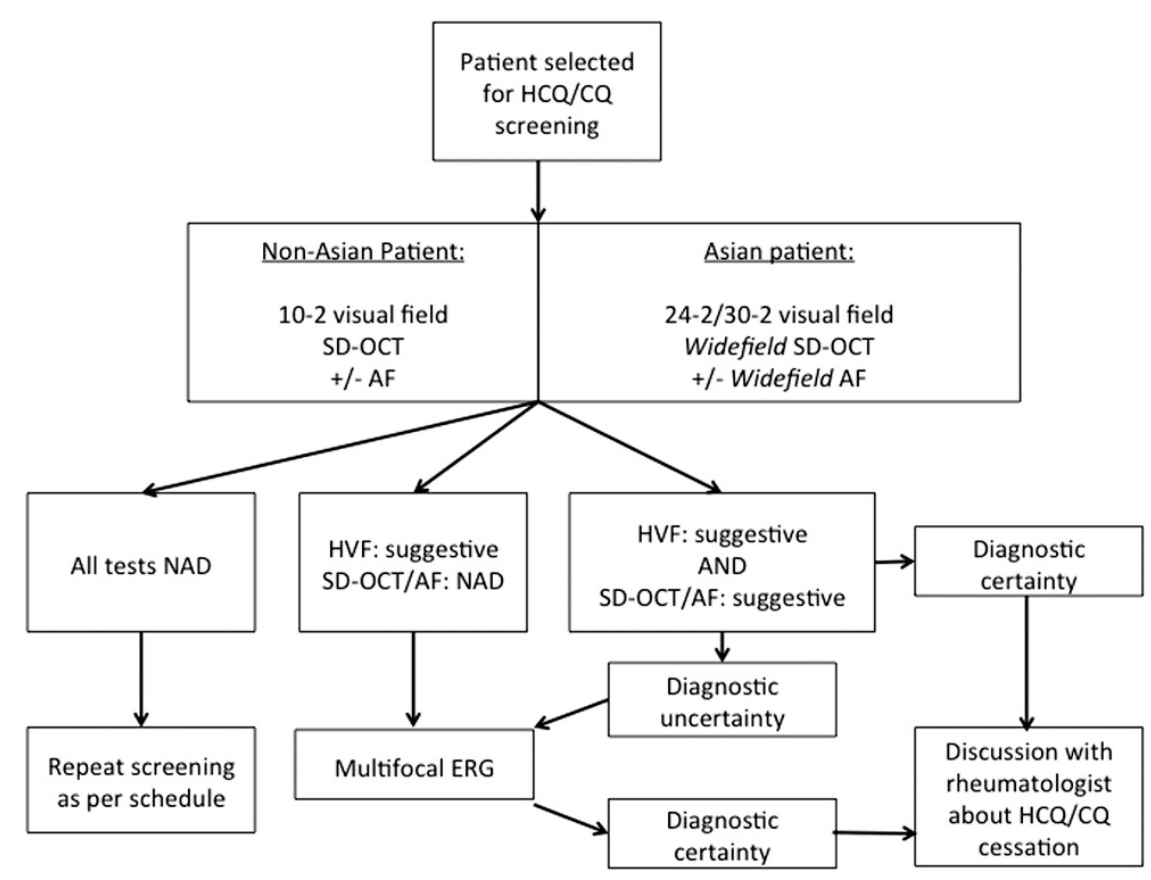

Figure 1 Suggested algorithm for the sequence of diagnostic tests performed during screening for HCQ/CQ retinopathy. 
guidance about the tests that Optometrists in this context should undertake (visual acuity, fundus examination, visual field examination etc.) Advice and practice differs in different centres. If the patient is referred for an Ophthalmic review, functional and image based screening is undertaken. Table 2 compares screening protocols, including what is suggested as a baseline assessment.

\section{Ongoing monitoring/ screening}

Ongoing monitoring or screening refers to continued, usually annual surveillance of an individual taking HCQ who is considered to be at risk of retinopathy in which a pre-designated protocol of investigations are undertaken. Table 2 highlights the differences between screening schedules for HCQ retinopathy. It is important that patients are aware that monitoring for HCQ retinopathy cannot prevent the occurrence of retinopathy but timely cessation of therapy at the earliest sign of any potential damage is expected to help limit either development or progression of loss of visual function. Patients taking HCQ/CQ must be informed about the risk of retinopathy by the prescribing physician, and the proposed schedule for baseline screening and ongoing monitoring for that individual taking into consideration their specific ocular and systemic risk factors. This should be in accordance with the most recent evidence and their current ocular and systemic status, which should be reviewed if there are changes in health and medication. ${ }^{25,28}$

More recent guidelines have favoured objective testing where possible (SD-OCT, AF, mfERG), ${ }^{25}$ although subjective testing (visual field analysis) remains of great value; data suggest that subtle scotomata on $10-2$ visual fields may be evident before structural changes are evident on SD-OCT (Figure 1). Guidelines suggest that at least one objective test should confirm findings on subjective testing before a diagnosis of HCQ retinopathy is made. Amsler grid testing, colour vision testing, fluorescein angiography, full-field ERG, time domain OCT and fundus photography have been removed from recommended protocols for screening as they are not considered sufficiently sensitive or specific to detect early disease. ${ }^{25}$ There are new technologies that may provide more sensitive objective structural (adaptive optics imaging), and functional (microperimetry) evaluation although these are essentially research tools at this time and not widely available, nor validated for screening purposes.

\section{Visual field testing: subjective, functional evaluation}

Automated threshold visual field analysis has become a key tool in the evaluation of patients at risk of HCQ retinopathy. Visual field analysis remains the most sensitive subjective investigation for the diagnosis of this disorder. The interpretation of scotomata may be challenging: whilst isolated points of reduction in sensitivity might be considered insignificant and more suggestive of patients without HCQ retinopathy, ${ }^{63}$ they may also represent the earliest evidence of HCQ retinopathy. ${ }^{25}$ Repeating visual field testing in this patient group is advisable. Contiguous scotoma points, and those identified in an annular zone between 2 and 8 degrees from fixation were found to be more likely to represent HCQ retinopathy in one study, with white targets considered more discriminating than red. ${ }^{63,64}$ Parafoveal scotomata are typical of HCQ retinopathy: superonasal scotomata are the most common abnormality as the inferotemporal paramacular region is often affected first. However, this observation is not absolute. ${ }^{25}$ Recent data have suggested that Asian patients should receive 30-2 or 24-2 visual field testing rather than 10-2, as wider-field visual field analysis is more likely to detect extramacular disease. ${ }^{62}$ Any central scotoma in 24-2 or 30-2 field testing should be taken seriously and further clarified with 10-2 visual fields. Any persistent or convincing scotoma should prompt evaluation by SD-OCT, AF or mfERG, with the mfERG particularly helpful where SD-OCT and AF do not detect objective evidence of HCQ toxicity suspected from visual field testing (Figure 1).

\section{Spectral-domain optical coherence tomography: objective, structural evaluation}

Spectral-domain OCT (SD-OCT) is a widely available technology that permits objective, structural evaluation of the macula in patients at risk of HCQ retinopathy. Typical findings of HCQ retinopathy on SD-OCT are parafoveal abnormalities of the outer segment, particularly thinning or loss of the photoreceptor layer, outer nuclear layer and/or ellipsoid zone (inner segment/ outer segment junction), and retinal pigment epithelium. ${ }^{65}$ These signs are considered strongly suggestive of HCQ toxicity. Focal disruption of outer segment structures is a typical finding of HCQ retinopathy, rather than widespread, progressive thinning. Asian eyes may manifest an extra-macular pattern of disease with SD-OCT abnormalities identified towards or beyond the vascular arcades: wide-field SD-OCT may be necessary for screening in this patient group. ${ }^{62}$

The inner retina did not demonstrate any abnormalities in early disease, in a study comparing SD-OCT images in both short term ( $<5$ years) and long term ( $>15$ years) users prior to the development of retinopathy. ${ }^{66}$ This is true both before and after drug cessation, ${ }^{39}$ although high cumulative doses may cause thinning of the ganglion cell/inner plexiform thickness. ${ }^{67}$ SD-OCT abnormalities 
persist on imaging after the cessation of $\mathrm{HCQ} / \mathrm{CQ}$, despite evidence of some visual field recovery. ${ }^{68}$

\section{Fundus auto-fluorescence: objective, structural evaluation}

Fundus autofluorescence (AF) using blue light of $488 \mathrm{~nm}$ wavelength, exploits the fluorescent property of lipofuscin within the retinal pigment epithelium (RPE). AF has been demonstrated to detect HCQ retinopathy, although was found to be less sensitive than mfERG. ${ }^{69} \mathrm{~A}$ parafoveal increased autofluorescence signal is detected in the early stages of disease, with mottled decrease in parafoveal autofluorescence as RPE degeneration becomes established in later stages of disease. ${ }^{69}$ AF may identify a sub-clinical bulls-eye maculopathy in patients with HCQ who demonstrate only fine RPE changes on fundoscopic examination, ${ }^{70}$ although modern screening techniques should identify HCQ before this stage. ${ }^{71}$ The wider, diffuse parafoveal autofluorescence signal seen in early disease is subtle, ${ }^{71}$ and quantitative

autofluorescence may increase the objectivity of this early feature. Increased signal on AF may precede SD-OCT features in this disorder. ${ }^{38}$ Widefield $\mathrm{AF}$ is required in Asian patients with suspected HCQ retinopathy to detect extramacular disease. ${ }^{62}$

\section{Multi-focal electroretinography: objective, functional evaluation}

Multifocal electroretinography produces a topographic representation of retinal responses and can identify local areas of depressed retinal sensitivity in early HCQ retinopathy. It has in some series been considered the gold standard test for the detection of suspected HCQ retinopathy. ${ }^{72}$ Comparing rings of responses about the foveal centre can increase the sensitivity of mfERG in detecting parafoveal disease. Asian patients will require wider-field mfERG for the purposes of screening; those extending to 20 degrees eccentricity in this patient group have been presented. ${ }^{62}$

MfERG has gained favour as more informed guidelines have emphasized the value of objective tests (OCT, mfERG, and AF), over subjective ones (visual field analysis). ${ }^{24,25} \mathrm{~A}$ recent systematic review suggested that mfERG may have the ability to detect HCQ retinopathy earlier than other tests used in HCQ screening (visual field, AF, and OCT; Table 3). ${ }^{73}$ However, screening guidelines must be practical to promote compliance across ophthalmic units; ${ }^{25}$ local access to electrodiagnostic testing is unlikely for the majority of HCQ patients undergoing screening. It is likely that mfERG will be indicated in patients with reproducible, suggestive visual field abnormalities in whom SD-OCT/AF imaging is unremarkable to establish objective evidence of HCQ retinopathy (Figure 1). Referral to a regional unit may be required to provide reliable electrodiagnostic testing in accordance with International Society for the Clinical Electrophysiology of Vision standards, which may involve visual evoked potentials, pattern and full-field electroretinography in addition to mfERG.

\section{Whether to implement a monitoring programme for all patients on $\mathrm{HCQ}$}

Screening for HCQ/CQ retinopathy has been advocated by the American Academy of Ophthalmology (AAO) since 2002. ${ }^{23-25,74}$ In the United Kingdom, the Royal College of Ophthalmologists, the British Society of Rheumatology and British Association of Dermatologists published joint guidelines in 2009 (updated from 1998 and 2004), prior to the revised AAO guidelines in 2011 and 2016. ${ }^{22}$ The United Kingdom guidelines advise against routine screening for HCQ toxicity. ${ }^{22}$ The panel's opinion was that the screening criteria were not met: in particular that the occurrence of 'clinically significant maculopathy was very rare' and that there is no reliable test for detecting it at a reversible stage. ${ }^{22}$ Under these guidelines, referral to an Ophthalmologist is recommended only when visual symptoms develop and are not correctable by an Optometrist. HCQ/CQ retinopathy is now considered far more common (overall prevalence of $7.5 \%$ of those patients taking the drug for 5 years or more, increasing to $20-50 \%$ after 20 years of therapy) ${ }^{28}$ than previous epidemiological studies suggested. These data suggest HCQ/CQ retinopathy is a significant public health problem. In addition, diagnostic tools required to detect $\mathrm{HCQ} / \mathrm{CQ}$ retinopathy at an early stage are now widely available (SD-OCT, visual field testing, $\mathrm{AF}$ ), and are proven to detect $\mathrm{HCQ} / \mathrm{CQ}$ toxicity before the onset of symptoms and fundus abnormalities are visible. ${ }^{24,25}$ We strongly recommend that these guidelines are reviewed and the advice against ongoing screening (monitoring) for HCQ/CQ retinopathy is updated to advise regular monitoring. The form that this should take needs to be the subject of revised guidelines.

Table 3 Sensitivity and specificity of diagnostic tests in HCQ retinopathy. ${ }^{68}$

\begin{tabular}{lcc}
\hline & Sensitivity & Specificity \\
\hline 10-2 VF & $85.7 \%$ & $92.5 \%$ \\
SD-OCT & $78.6 \%$ & $86.9 \%$ \\
Multifocal ERG & $92.9 \%$ & $98.1 \%$ \\
SD-OCT and 10-2 VF & $85.7 \%$ & $92.5 \%$ \\
10-2 VF and mfERG & $100 \%$ & $82.2 \%$ \\
SD-OCT and mfERG & $100 \%$ & $86.0 \%$ \\
\hline
\end{tabular}




\section{Screening schedule}

Most screening guidelines recommend screening for HCQ/CQ retinopathy after 5 years of drug therapy (Table 2) with annual screening. Baseline evaluation, in some form, is widely recommended to identify those with pre-existing maculopathy and to establish baseline measures from which to identify change. In patients with unusual risk factors, screening may be indicated before 5 years (Table 2) ${ }^{25}$ The duration of screening is not specified by most guidelines but is likely to be required indefinitely, particularly as new data suggest prevalence of toxicity in those with greater than 20 years of therapy to be as high as $20-50 \% .^{28}$

\section{Screening Protocol: How to screen/monitor}

Screening/Monitoring for HCQ should use diagnostic instruments that are widely available with easily interpretable images to ensure compliance with screening guidelines. Visual field analysis (10-2, 24-2 or 30-2 as appropriate), and SD-OCT are widely available and the consensus is emerging that they should, in combination, be performed on every patient presenting for HCQ screening. One study identified ring scotomata on visual field testing with normal SD-OCT appearance was present in $10 \%$ of patients; conversely, all patients with SD-OCT abnormalities had scotomata on visual field testing thus suggesting that visual field testing is a sensitive test to elicit early signs of toxicity. ${ }^{75}$

Fundus autofluorescence (AF) may be a useful additional tool to provide objective, structural evidence of disease that may correlate with findings on SD-OCT imaging and visual field analysis. AF imaging (wide-field AF in Asian patients) should be considered in HCQ patients where available.

Multifocal ERG may provide objective, functional evidence of disease, and may be useful when the diagnosis of HCQ is uncertain: in particular when visual field analysis demonstrates scotomata in the absence of structural evidence on SD-OCT or AF.

The efficacy of screening is dependent on the relative sensitivity and specificity of screening tests used to detect pre-symptomatic disease. One study found the sensitivities of 10-2 HVF, mfERG and SD-OCT in detected HCQ retinopathy were $85.7,92.9$, and $78.6 \%$ respectively, with specificities of $92.5,86.9$, and $98.1 \%$ (Table 3 ). ${ }^{76}$

Negative predictive values were greater than $99 \%$ for all tests: confident exclusion of retinopathy is possible when a combination of diagnostic tests are negative. However, when isolated tests are positive, they are less useful in predicting disease: the positive predictive value may be as low as 30\% for some diagnostic tests (such as 10-2 HVF, mfERG and SD-OCT) in HCQ/CQ retinopathy. ${ }^{76}$
Figure 1 presents a suggested algorithm for the interpretation of diagnostic tests in patients with HCQ/ $\mathrm{CQ}$ who are referred for screening.

\section{Cost of screening}

The economic cost of providing HCQ screening in any country will depend on the number of individuals in the population, the total number of individuals taking HCQ, and the prevalence of risk factors for screening within that population of HCQ users. Screening may involve at least an annual ophthalmological assessment for patients taking HCQ/CQ and may be indefinite whilst the patient remains on the drug. ${ }^{25} \mathrm{HCQ}$ screening may require additional outpatient capacity for diagnostic tests, and training on their interpretation: this may take place in virtual clinics in which the tests are performed by technicians and interpreted by those trained to do so. MfERG will usually need a referral to a regional unit if required. If the decision to institute screening for HCQ is established, a full economic evaluation will be required on a national level to determine if screening for HCQ is costeffective.

When reviewing the need to implement screening for HCQ/CQ retinopathy, the potential drawbacks should be considered. It may cause harm: anxiety to patient groups potentially at risk of retinopathy; and false positive results may lead to inappropriately stopping HCQ - an effective drug with a demonstrated survival benefit. It is likely that incidental ocular co-pathology will be detected through any screening programme and require further investigation and/or treatment.

\section{Adherence to screening}

Adherence to published guidelines on HCQ/CQ retinopathy screening is challenging. One study determined that only $54.8 \%$ of patients received appropriate evaluation in a US cohort, when audited against the recommendations of the AAO (this study was published in 2015, 4 years after the updated AAO guidelines, published 2011). In this study, $25.7 \%$ received fewer than the recommended investigations; SD-OCT and 10-2 visual field testing were often performed, but AF and mfERG often omitted. ${ }^{77}$

Should a screening programme for HCQ retinopathy be instituted, additional administrative support and regional organisation, similar to that used to organise retinal screening for patients with diabetes in the UK, may be required to introduce quality control into the process of screening: ensuring patients are assessed, and recalled when required. The caring physician - either Rheumatologist or General Practitioner - and patients on 
HCQ will need to be aware of the schedule for screening so that patients requiring screening receive it.

\section{Management}

There is no currently proven effective treatment for visual restoration in patients with HCQ/CQ retinopathy. Management centres around identification of definite HCQ/CQ retinopathy, drug cessation in consultation with a rheumatologist and visual rehabilitation as required.

\section{Hydroxychloroquine drug cessation}

The decision to stop HCQ/CQ therapy will depend on the certainty of toxic retinopathy: the definitions of 'possible', 'likely', and 'definite' HCQ/CQ retinopathy would be helpful in guiding discussions with patient and Rheumatologist about whether drug cessation with a switch to alternative treatment is recommended, or HCQ/CQ continued while further testing is awaited. ${ }^{24}$ Given the long half-life of $\mathrm{HCQ} / \mathrm{CQ}^{12}$, systemic clearance is delayed for months after drug cessation, and some degree of ongoing toxicity is likely to continue for this period. ${ }^{78}$ It has been suggested that the continuing toxic effects following drug cessation are likely to be related to the severity of toxic maculopathy at the time HCQ/CQ was stopped. ${ }^{25,59}$ One longitudinal study evaluated visual acuity, SD-OCT imaging data and mfERG of patients on HCQ, and 6 months following drug cessation finding improved visual acuity and mfERG responses, but not OCT appearances. ${ }^{61} \mathrm{~A}$ further study evaluating only SDOCT imaging characteristics before and for 4 years after drug cessation in 11 patients with HCQ retinopathy found that if HCQ is stopped before RPE damage, progression of disease on SD-OCT is limited to the first year and diffuse in distribution without parafoveal localisation. ${ }^{39}$ There is some evidence that preservation of the external limiting membrane is a favourable prognostic sign in HCQ retinopathy. ${ }^{78}$

\section{Role of the Rheumatologist}

The clinical decision to initiate or stop treatment with $\mathrm{HCQ}$, or to change the treatment dose is likely to be made by a Rheumatologist. It is important that Rheumatologists are aware of the updated evidence that ABW (less than $5.0 \mathrm{mg} / \mathrm{kg}$ of $\mathrm{ABW} /$ day) should be prescribed to patients as current best practice, and is more strongly correlated with retinal toxicity than IBW when prescribing HCQ. ${ }^{25,28}$ Similarly, emerging risk factors for HCQ/CQ retinopathy and updated screening schedules (Table 2) may prompt screening before 5 years of drug therapy (ie, Tamoxifen use). ${ }^{25}$ Rheumatologists are likely to be the most common route of referral of at risk patients taking HCQ who require screening to Ophthalmologists: patients in a US study cared for by a rheumatologist had a greater likelihood of regular eye screening. ${ }^{79}$

\section{Future directions}

\section{Prevention of retinal toxicity}

The most important means of reducing harm from HCQ/ $\mathrm{CQ}$ is accurate dosing based on current data on retinal toxicity using $\mathrm{ABW}$ at less than $5.0 \mathrm{mg} / \mathrm{kg}$ of $\mathrm{ABW} /$ day rather than IBW at a dose of less than $6.5 \mathrm{mg} / \mathrm{kg}$ of IBW/ day. However, in certain patients such as those who are obese, very short or very thin, there may be a significant difference between these ABW and IBW dose calculations. ${ }^{25,28}$ Electronic prescribing systems, and more rigorous and universal prescribing protocols for HCQ may prevent cases of retinal toxicity. All prescribing clinicians should ensure dosing is optimised, and rechecked if large changes in weight or renal function occur. One retrospective audit found that of 675 patients treated with HCQ in a single centre, around $46 \%$ of males and $56 \%$ of females were receiving higher doses of HCQ than recommended. ${ }^{42}$ In our audit, approximately $10 \%$ of patients were overdosed when ABW was used and 30\% when IBW was used to calculate optimal HCQ dose based on conventional dosing recommendations of less than $6.5 \mathrm{mg} / \mathrm{kg} /$ day (unpublished data). Accurate dosing of $\mathrm{HCQ} / \mathrm{CQ}$ may reduce the risk of toxicity on a population level.

\section{Serum hydroxychloroquine monitoring}

Serum monitoring of HCQ may be used in patients with renal or hepatic dysfunction who may exhibit variable metabolism or excretion of HCQ, or to differentiate treatment compliance and treatment failure. ${ }^{80,81}$ In some cases, sub-therapeutic serum HCQ concentrations were due to a lower than optimal dose, and higher doses associated with fewer flares in patients in remission. ${ }^{81}$ The large, randomized, placebo-controlled clinical trial (PLUS study) with 573 patients with SLE found that although low serum concentration of HCQ was associated with increased disease activity in SLE, it did not translate to disease flares over a 7-month follow-up period. ${ }^{82} \mathrm{HCQ}$ dosing may therefore reflect a balance between short-term systemic efficacy and long-term ocular safety. Serum HCQ monitoring has not demonstrated any clear role in predicting the risk of retinopathy in any published study. 


\section{Objective, structural evaluation: adaptive optics imaging}

Adaptive optics (AO) technology permits the evaluation of photoreceptor density and morphology. ${ }^{83}$ Interest has been expressed in the potential of this technology to detect very early structural abnormalities in HCQ retinopathy, ${ }^{25}$ particularly since the photoreceptor layer is considered the primary site of toxicity. AO technology has identified patch cone mosaic lesions in which cone photoreceptors were either missing or lost with atypical morphology of residual cones in two patients with symptomatic HCQ retinopathy. ${ }^{84}$ This structural deficit corresponded to scotomata identified on visual field testing. OCT appearances have been correlated with photoreceptor appearances using $\mathrm{AO}$ technology in various maculopathies, ${ }^{83}$ and it is suggested that the relationship between outer retinal reflectivity on SD-OCT and photoreceptor density on adaptive optics imaging may be helpful. ${ }^{85} \mathrm{AO}$ technology may find a role in providing objective structural evidence of disease in the early detection of HCQ retinopathy. A larger series correlating the structural changes on AO technology with functional tools such as mfERG and visual field analysis is required to evaluate the sensitivity and specificity of $\mathrm{AO}$ imaging. The limited availability of this technology is likely to exclude it from any screening protocols in the near future, although it may be useful as a research tool in cases of suspected HCQ retinopathy. ${ }^{86}$

\section{Subjective, functional evaluation: Microperimetry}

Microperimetry is a technique of visual field testing where light stimuli are projected onto specific points on a subject's retina at which the threshold of retinal sensitivity is established. Reduced overall macular sensitivity on microperimetry was detected in patients taking HCQ versus healthy controls, $, 8,88$ but no difference in foveal sensitivity was detected in one study. ${ }^{88}$ A further study also identified reduced overall macular sensitivity in patients taking HCQ despite normal mfERG, 10-2 visual fields, OCT and AF. ${ }^{89}$ The role of microperimetry in HCQ screening is as yet unknown; ${ }^{90}$ further studies are required to determine the specificity and sensitivity of microperimetry in suspected HCQ retinopathy, and whether it exceeds that of standard automated visual field testing.

\section{Conclusion}

This subject of this review article is chloroquine and HCQ retinopathy and it is clear that with long-term use and in susceptible patients that some patients may develop severe visual loss. What is emerging from the literature over the last few years, and has been incorporated into the latest AAO 2016 guidelines, is that regular monitoring is important when using this extremely useful medication. In addition, those involved in the care of patients taking this drug need to be aware of accurate dosing by using the ABW, and to take particular care in obese, or short individuals. Concomitant medication may have an additive deleterious effect; and that long term use, particularly in the elderly is associated with retinopathy in a significant number of patients. Emerging data have shown a prevalence of HCQ retinopathy of $7.5 \%$ in those taking the drug for greater than 5 years long-term HCQ users with between 20 and 50\% for those who have taken it over 20 years. ${ }^{43}$ The knowledge that tamoxifen use has emerged as a significant risk factor and that there may be variable expression of disease according to race ${ }^{62}$ suggests that a review of the RCOphth joint guidelines on HCQ screening should be considered. The AAO guidelines in 2011 suggested a shift towards more objective tests of visual function (SD-OCT, mfERG or AF), in addition to subjective tests (10-2 visual field testing), ${ }^{24}$ and the recent updated AAO guidelines (2016) acknowledged that a more realistic protocol might improve compliance within ophthalmic units. ${ }^{25}$ Automated visual field testing, together with SD-OCT and AF, should be achievable and practical for most ophthalmic units; those requiring mfERG testing could be referred to a regional centre. Clinical criteria to define possible, likely or definite HCQ toxicity on the basis of diagnostic testing would be helpful for both the general Ophthalmologist to interpret diagnostic results, and to the Rheumatologist to determine whether HCQ cessation is justified, particularly given a proven survival benefit of HCQ therapy has been identified in patients SLE. $^{2}$

A national case-finding study evaluating the incidence of HCQ retinopathy would assist greatly in understanding the burden of disease and modelling the cost-effectiveness of any screening programme; there are limitations on the inferences that can be made from cohort studies and across study populations. Ophthalmologists, Rheumatologists and General Practitioners should be aware of the best practice in safe prescribing of HCQ, and any consensus screening criteria to ensure those who require screening for HCQ retinopathy are appropriately referred. Audit of ophthalmic screening practice will be required to ensure that the recommendations for screening are being met. We initiated an audit of our current service as a consequence of identifying 3 patients on long-term HCQ who developed HCQ retinopathy (Latasiewicz et al ${ }^{91}$ ) and have implemented change as a consequence of this (unpublished data). Patient education about the risks of visual loss with HCQ or CQ therapy is crucial: risks of visual loss may be minimized, but not altogether eliminated, with regular ophthalmic screening. Original epidemiological data identifying the prevalence 
of HCQ retinopathy in 7.5\% of long-term HCQ users may now suggest a medico-legal imperative to screen those patients considered at high risk of HCQ retinopathy. In addition in this review article we have proposed a practical protocol for screening these patients to detect any sign of HCQ retinopathy as early as possible.

\section{Methodology}

We used the following databases and search terms to research this review: MEDLINE/PubMed:

'Hydroxychloroquine retinopathy'; 'Hydroxychloroquine maculopathy'; 'Hydroxychloroquine [title], SLE';

'Chloroquine retinopathy'; Original research studies; Non-English papers excluded.

\section{Conflict of interest}

The authors declare no conflict of interest.

\section{References}

1 Gabourel JD. Effects of hydroxychloroquine on the growth of mammalian cells in vitro. J Pharmacol Exp Ther 1963; 141: 122-130.

2 Alarcon GS, McGwin G, Bertoli AM, Fessler BJ, Calvo-Alen J, Bastian HM et al. Effect of hydroxychloroquine on the survival of patients with systemic lupus erythematosus: data from LUMINA, a multiethnic US cohort (LUMINA L). Ann Rheum Dis 2007; 66: 1168-1172.

3 Pons-Estel GJ, Alarcon GS, McGwin Jr G, Danila MI, Zhang J, Bastian HM et al. Protective effect of hydroxychloroquine on renal damage in patients with lupus nephritis: $\mathrm{LXV}$, data from a multiethnic US cohort. Arthritis Rheum 2009; 61: 830-839.

4 Willis R, Seif AM, McGwin Jr G, Martinez-Martinez LA, Gonzalez EB, Dang N et al. Effect of hydroxychloroquine treatment on pro-inflammatory cytokines and disease activity in SLE patients: data from LUMINA (LXXV), a multiethnic US cohort. Lupus 2012; 21: 830-835.

5 Costedoat-Chalumeau N, Dunogue B, Morel N, Le Guern V, Guettrot-Imbert G. Hydroxychloroquine: a multifaceted treatment in lupus. Presse Med 2014; 43: e167-e180.

6 Jancinova V, Pazourekova S, Lucova M, Perecko T, Mihalova D, Bauerova $\mathrm{K}$ et al. Selective inhibition of extracellular oxidants liberated from human neutrophils-A new mechanism potentially involved in the antiinflammatory activity of hydroxychloroquine. Int Immunopharmacol 2015; 28: 175-181.

7 Goldman FD, Gilman AL, Hollenback C, Kato RM, Premack BA, Rawlings DJ. Hydroxychloroquine inhibits calcium signals in T cells: a new mechanism to explain its immunomodulatory properties. Blood 2000; 95: 3460-3466.

8 van Loosdregt J, Spreafico R, Rossetti M, Prakken BJ, Lotz M, Albani S. Hydroxychloroquine preferentially induces apoptosis of CD45RO+ effector T cells by inhibiting autophagy: a possible mechanism for therapeutic modulation of T cells. J Allergy Clin Immunol 2013; 131: 1443-6.e1.

9 Fox RI. Mechanism of action of hydroxychloroquine as an antirheumatic drug. Semin Arthritis Rheum 1993; 23: 82-91.
10 Scholl HP, Shah SM. We need to be better prepared for hydroxychloroquine retinopathy. JAMA Ophthalmol 2014; 132: 1460-1461.

11 Bethel M, Yang FM, Li S, Nahman NS, Oliver AM, Machua W et al. Hydroxychloroquine in patients with systemic lupus erythematosus with end-stage renal disease. J Investig Med 2016; 64: 908-910.

12 Electronic Medicines Compendium. Plaquenil 200mg Filmcoated Tablets. 2014.

13 Ochsendorf FR. Use of antimalarials in dermatology. J Dtsch Dermatol Ges 2010; 8: 829-844 quiz 45.

14 Braun S, Ferner M, Kronfeld K, Griese M. Hydroxychloroquine in children with interstitial (diffuse parenchymal) lung diseases. Pediatr Pulmonol 2015; 50: 410-419.

15 Poklepovic A, Gewirtz DA. Outcome of early clinical trials of the combination of hydroxychloroquine with chemotherapy in cancer. Autophagy 2014; 10: 1478-1480.

16 Cook KL, Warri A, Soto-Pantoja DR, Clarke PA, Cruz MI, Zwart A et al. Hydroxychloroquine inhibits autophagy to potentiate antiestrogen responsiveness in ER+ breast cancer. Clin Cancer Res 2014; 20: 3222-3232.

17 Goldberg SB, Supko JG, Neal JW, Muzikansky A, Digumarthy S, Fidias $\mathrm{P}$ et al. A phase I study of erlotinib and hydroxychloroquine in advanced non-small-cell lung cancer. J Thorac Oncol 2012; 7: 1602-1608.

18 Mahalingam D, Mita M, Sarantopoulos J, Wood L, Amaravadi RK, Davis LE et al. Combined autophagy and HDAC inhibition: a phase I safety, tolerability, pharmacokinetic, and pharmacodynamic analysis of hydroxychloroquine in combination with the HDAC inhibitor vorinostat in patients with advanced solid tumors. Autophagy 2014; 10: 1403-1414.

19 Rangwala R, Chang YC, Hu J, Algazy KM, Evans TL, Fecher LA et al. Combined MTOR and autophagy inhibition: phase I trial of hydroxychloroquine and temsirolimus in patients with advanced solid tumors and melanoma. Autophagy 2014; 10: 1391-1402.

20 Rangwala R, Leone R, Chang YC, Fecher LA, Schuchter LM, Kramer A et al. Phase I trial of hydroxychloroquine with doseintense temozolomide in patients with advanced solid tumors and melanoma. Autophagy 2014; 10: 1369-1379.

21 Vogl DT, Stadtmauer EA, Tan KS, Heitjan DF, Davis LE, Pontiggia L et al. Combined autophagy and proteasome inhibition: a phase 1 trial of hydroxychloroquine and bortezomib in patients with relapsed/refractory myeloma. Autophagy 2014; 10: 1380-1390.

22 The Royal College of Ophthalmologists. Hydroxychloroquine and Ocular ToxicityRecommendations on Screening, 2009.

23 Marmor MF, Carr RE, Easterbrook M, Farjo AA, Mieler WF. Recommendations on screening for chloroquine and hydroxychloroquine retinopathy: a report by the American Academy of Ophthalmology. Ophthalmology 2002; 109: 1377-1382.

24 Marmor MF, Kellner U, Lai TY, Lyons JS, Mieler WF. Revised recommendations on screening for chloroquine and hydroxychloroquine retinopathy. Ophthalmology 2011; 118: 415-422.

25 Marmor MF, Kellner U, Lai TY, Melles RB, Mieler WF. Recommendations on Screening for Chloroquine and Hydroxychloroquine Retinopathy (2016 Revision) Ophthalmology 2016; 123: 1386-1394.

26 Rainsford KD, Parke AL, Clifford-Rashotte M, Kean WF. Therapy and pharmacological properties of 
hydroxychloroquine and chloroquine in treatment of systemic lupus erythematosus, rheumatoid arthritis and related diseases. Inflammopharmacology 2015; 23: 231-269.

27 Leroux M, Desveaux C, Parcevaux M, Julliac B, Gouyon JB, Dallay $\mathrm{D}$ et al. Impact of hydroxychloroquine on preterm delivery and intrauterine growth restriction in pregnant women with systemic lupus erythematosus: a descriptive cohort study. Lupus 2015; 24: 1384-1391.

28 Melles RB, Marmor MF. The risk of toxic retinopathy in patients on long-term hydroxychloroquine therapy. JAMA Ophthalmol 2014; 132: 1453-1460.

29 Giner Galvan V, Oltra MR, Rueda D, Esteban MJ, Redon J. Severe acute hepatitis related to hydroxychloroquine in a woman with mixed connective tissue disease. Clin Rheumatol 2007; 26: 971-972.

30 Makin AJ, Wendon J, Fitt S, Portmann BC, Williams R. Fulminant hepatic failure secondary to hydroxychloroquine. Gut 1994; 35: 569-570.

31 Petri M. Use of hydroxychloroquine to prevent thrombosis in systemic lupus erythematosus and in antiphospholipid antibody-positive patients. Curr Rheumatol Rep 2011; 13: $77-80$.

32 Sauer BC, Teng CC, Tang D, Leng J, Curtis JR, Mikuls TR et al. Persistence with conventional triple therapy versus a tumor necrosis factor inhibitor and methotrexate in U.S. veterans with rheumatoid arthritis. Arthritis Care Res (Hoboken) 2017; 69: 313-322.

33 Hazlewood GS, Barnabe C, Tomlinson G, Marshall D, Devoe D, Bombardier C. Methotrexate monotherapy and methotrexate combination therapy with traditional and biologic disease modifying antirheumatic drugs for rheumatoid arthritis: abridged Cochrane systematic review and network meta-analysis. BMJ 2016; 353: i1777.

34 Rantalaiho V, Puolakka K, Korpela M, Hannonen P, Mottonen T. Long-term results of the FIN-RACo trial; treatment with a combination of traditional diseasemodifying anti-rheumatic drugs is an excellent option in early rheumatoid arthritis. Clin Exp Rheumatol 2012; 30: S27-S31.

35 Xu C, Zhu L, Chan T, Lu X, Shen W, Madigan MC et al. Chloroquine and hydroxychloroquine are novel inhibitors of human organic anion transporting polypeptide 1A2. J Pharm Sci 2016; 105: 884-890.

36 Korthagen NM, Bastiaans J, van Meurs JC, van Bilsen K, van Hagen PM, Dik WA. Chloroquine and Hydroxychloroquine Increase Retinal Pigment Epithelial Layer Permeability. J Biochem Mol Toxicol 2015; 29: 299-304.

37 Rosenthal AR, Kolb H, Bergsma D, Huxsoll D, Hopkins JL. Chloroquine retinopathy in the rhesus monkey. Invest Ophthalmol Vis Sci 1978; 17: 1158-1175.

38 Marmor MF. Comparison of screening procedures in hydroxychloroquine toxicity. Arch Ophthalmol 2012; 130: 461-469.

39 de Sisternes L, Hu J, Rubin DL, Marmor MF. Localization of damage in progressive hydroxychloroquine retinopathy on and off the drug: inner versus outer retina, parafovea versus peripheral fovea. Invest Ophthalmol Vis Sci 2015; 56: 3415-3426.

40 Bergholz R, Schroeter J, Ruther K. Evaluation of risk factors for retinal damage due to chloroquine and hydroxychloroquine. Br J Ophthalmol 2010; 94: 1637-1642.

41 Browning DJ, Lee C, Rotberg D. The impact of different algorithms for ideal body weight on screening for hydroxychloroquine retinopathy in women. Clin Ophthalmol 2014; 8: 1401-1407.

42 Walvick MD, Walvick MP, Tongson E, Ngo CH. Hydroxychloroquine: lean body weight dosing. Ophthalmology 2011; 118: 2100.

43 Wolfe F, Marmor MF. Rates and predictors of hydroxychloroquine retinal toxicity in patients with rheumatoid arthritis and systemic lupus erythematosus. Arthritis Care Res (Hoboken) 2010; 62: 775-784.

44 Falcone PM, Paolini L, Lou PL. Hydroxychloroquine toxicity despite normal dose therapy. Ann Ophthalmol 1993; 25: $385-388$.

45 Cavagna L, Rossi P, Bogliolo L, Antoniazzi E, Gelmi C, Caporali R et al. [Early electroretinografic changes in elderly RA patients treated with hydroxychloroquine]. Reumatismo 2002; 54: 226-231.

46 Jallouli M, Galicier L, Zahr N, Aumaitre O, Frances C, Le Guern $\mathrm{V}$ et al. Determinants of hydroxychloroquine blood concentration variations in systemic lupus erythematosus. Arthritis Rheumatol 2015; 67: 2176-2184.

47 Nayfield SG, Gorin MB. Tamoxifen-associated eye disease. A review. J Clin Oncol 1996; 14: 1018-1026.

48 Kim LA, Amarnani D, Gnanaguru G, Tseng WA, Vavvas DG, D'Amore PA. Tamoxifen toxicity in cultured retinal pigment epithelial cells is mediated by concurrent regulated cell death mechanisms. Invest Ophthalmol Vis Sci 2014; 55: 4747-4758.

49 Toimela T, Tahti H, Salminen L. Retinal pigment epithelium cell culture as a model for evaluation of the toxicity of tamoxifen and chloroquine. Ophthalmic Res 1995; 27(Suppl 1): 150-153.

50 Leung LS, Neal JW, Wakelee HA, Sequist LV, Marmor MF. Rapid onset of retinal toxicity from high-dose hydroxychloroquine given for cancer therapy. Am J Ophthalmol 2015; 160: 799-805.e1.

51 Navajas EV, Krema H, Hammoudi DS, Lipton JH, Simpson ER, Boyd $S$ et al. Retinal toxicity of high-dose hydroxychloroquine in patients with chronic graft-versus-host disease. Can J Ophthalmol 2015; 50: 442-450.

52 Anderson NG, Regillo C. Ocular manifestations of graft versus host disease. Curr Opin Ophthalmol 2004; 15: 503-507.

53 Shroyer NF, Lewis RA, Lupski JR. Analysis of the ABCR (ABCA4) gene in 4-aminoquinoline retinopathy: is retinal toxicity by chloroquine and hydroxychloroquine related to Stargardt disease? Am J Ophthalmol 2001; 131: 761-766.

54 Grassmann F, Bergholz R, Mandl J, Jagle H, Ruether K, Weber BH. Common synonymous variants in ABCA4 are protective for chloroquine induced maculopathy (toxic maculopathy). BMC Ophthalmol 2015; 15: 18.

55 Maugeri A, Flothmann K, Hemmrich N, Ingvast S, Jorge P, Paloma E et al. The ABCA4 2588G > C Stargardt mutation: single origin and increasing frequency from South-West to North-East Europe. Eur J Hum Genet 2002; 10: 197-203.

56 Lambertus S, van Huet RA, Bax NM, Hoefsloot LH, Cremers FP, Boon CJ et al. Early-onset stargardt disease: phenotypic and genotypic characteristics. Ophthalmology 2015; 122: 335-344.

57 Noupuu K, Lee W, Zernant J, Greenstein VC, Tsang S, Allikmets R. Recessive Stargardt disease phenocopying hydroxychloroquine retinopathy. Graefes Arch Clin Exp Ophthalmol 2015; 254: 865-872.

58 Mavrikakis I, Sfikakis PP, Mavrikakis E, Rougas K, Nikolaou A, Kostopoulos C et al. The incidence of irreversible retinal toxicity in patients treated with 
hydroxychloroquine: a reappraisal. Ophthalmology 2003; 110: 1321-1326.

59 Kellner S, Weinitz S, Farmand G, Kellner U. Cystoid macular oedema and epiretinal membrane formation during progression of chloroquine retinopathy after drug cessation. Br J Ophthalmol 2014; 98: 200-206.

60 Brinkley Jr JR, Dubois EL, Ryan SJ. Long-term course of chloroquine retinopathy after cessation of medication. Am J Ophthalmol 1979; 88: 1-11.

61 Moschos MM, Nitoda E, Chatziralli IP, Gatzioufas Z, Koutsandrea C, Kitsos G. Assessment of hydroxychloroquine maculopathy after cessation of treatment: an optical coherence tomography and multifocal electroretinography study. Drug Des Devel Ther 2015; 9: 2993-2999.

62 Melles RB, Marmor MF. Pericentral retinopathy and racial differences in hydroxychloroquine toxicity. Ophthalmology 2015; 122: 110-116.

63 Browning DJ, Lee C. Scotoma analysis of 10-2 visual field testing with a white target in screening for hydroxychloroquine retinopathy. Clin Ophthalmol 2015; 9: 943-952.

64 Browning DJ, Lee C. Scotoma analysis of 10-2 visual field testing with a red target in screening for hydroxychloroquine retinopathy. Clin Ophthalmol 2015; 9: 1499-1509.

65 Rodriguez-Padilla JA, Hedges TR 3rd, Monson B, Srinivasan V, Wojtkowski M, Reichel E et al. High-speed ultra-high-resolution optical coherence tomography findings in hydroxychloroquine retinopathy. Arch Ophthalmol 2007; 125: 775-780.

66 de Sisternes L, Hu J, Rubin DL, Marmor MF. Analysis of inner and outer retinal thickness in patients using hydroxychloroquine prior to development of retinopathy. JAMA Ophthalmol; e-pub ahead of print 17 March 2016; doi:10.1001/jamaophthalmol.2016.0155.

67 Lee MG, Kim SJ, Ham DI, Kang SW, Kee C, Lee J et al. Macular retinal ganglion cell-inner plexiform layer thickness in patients on hydroxychloroquine therapy. Invest Ophthalmol Vis Sci 2014; 56: 396-402.

68 Turgut B, Turkcuoglu P, Serdar Koca S, Aydemir O. Detection of the regression on hydroxychloroquine retinopathy in optical coherence tomography. Clin Rheumatol 2009; 28: 607-609.

69 Kellner U, Renner AB, Tillack H. Fundus autofluorescence and mfERG for early detection of retinal alterations in patients using chloroquine/hydroxychloroquine. Invest Ophthalmol Vis Sci 2006; 47: 3531-3538.

70 Gorovoy IR, Gorovoy MS. Fundus autofluorescence in hydroxychloroquine toxicity. JAMA Ophthalmol 2013; 131: 506.

71 Marmor MF. Fundus autofluorescence is not the best early screen for hydroxychloroquine toxicity. JAMA Ophthalmol 2013; 131: 1487-1488.

72 Cukras C, Huynh N, Vitale S, Wong WT, Ferris FL 3rd, Sieving PA. Subjective and objective screening tests for hydroxychloroquine toxicity. Ophthalmology 2015; 122: 356-366.

73 Tsang AC, Ahmadi Pirshahid S, Virgili G, Gottlieb CC, Hamilton J, Coupland SG. Hydroxychloroquine and chloroquine retinopathy: a systematic review evaluating the multifocal electroretinogram as a screening test. Ophthalmology 2015; 122: 1239-51.e4.
74 Marmor MF. New American Academy of Ophthalmology recommendations on screening for hydroxychloroquine retinopathy. Arthritis Rheum 2003; 48: 1764.

75 Marmor MF, Melles RB. Disparity between visual fields and optical coherence tomography in hydroxychloroquine retinopathy. Ophthalmology 2014; 121: 1257-1262.

76 Browning DJ, Lee C. Relative sensitivity and specificity of 10-2 visual fields, multifocal electroretinography, and spectral domain optical coherence tomography in detecting hydroxychloroquine and chloroquine retinopathy. Clin Ophthalmol 2014; 8: 1389-1399.

77 Au A, Parikh V, Modi YS, Ehlers JP, Schachat AP, Singh RP. Hydroxychloroquine screening practice patterns within a large multispecialty ophthalmic practice. Am J Ophthalmol 2015; 160: 561-8, e2.

78 Mititelu M, Wong BJ, Brenner M, Bryar PJ, Jampol LM, Fawzi AA. Progression of hydroxychloroquine toxic effects after drug therapy cessation: new evidence from multimodal imaging. JAMA Ophthalmol 2013; 131: 1187-1197.

79 Nika M, Blachley TS, Edwards P, Lee PP, Stein JD. Regular examinations for toxic maculopathy in long-term chloroquine or hydroxychloroquine users. JAMA Ophthalmol 2014; 132: 1199-1208.

80 Durcan L, Clarke WA, Magder LS, Petri M. Hydroxychloroquine blood levels in systemic lupus erythematosus: clarifying dosing controversies and improving adherence. J Rheumatol 2015; 42: 2092-2097.

81 Mok CC, Penn HJ, Chan KL, Tse SM, Langman LJ, Jannetto PJ. Hydroxychloroquine serum concentrations and flares of systemic lupus erythematosus: A longitudinal cohort analysis. Arthritis Care Res (Hoboken) 2016; 68: 1295-1302.

82 Costedoat-Chalumeau N, Galicier L, Aumaitre O, Frances C, Le Guern V, Liote F et al. Hydroxychloroquine in systemic lupus erythematosus: results of a French multicentre controlled trial (PLUS Study). Ann Rheum Dis 2013; 72: 1786-1792.

83 Jacob J, Paques M, Krivosic V, Dupas B, Couturier A, Kulcsar C et al. Meaning of visualizing retinal cone mosaic on adaptive optics images. Am J Ophthalmol 2015; 159: 118-23.e1.

84 Bae EJ, Kim KR, Tsang SH, Park SP, Chang S. Retinal damage in chloroquine maculopathy, revealed by high resolution imaging: a case report utilizing adaptive optics scanning laser ophthalmoscopy. Korean J Ophthalmol 2014; 28: $100-107$.

85 Flores M, Debellemaniere G, Bully A, Meillat M, Tumahai P, Delbosc B et al. Reflectivity of the outer retina on spectraldomain optical coherence tomography as a predictor of photoreceptor cone density. Am J Ophthalmol 2015; 160: 588-95, e2.

86 Stepien KE, Han DP, Schell J, Godara P, Rha J, Carroll J. Spectral-domain optical coherence tomography and adaptive optics may detect hydroxychloroquine retinal toxicity before symptomatic vision loss. Trans Am Ophthalmol Soc 2009; 107: 28-33.

87 Martinez-Costa L, Victoria Ibanez M, Murcia-Bello C, Epifanio I, Verdejo-Gimeno C, Beltran-Catalan E et al. Use of microperimetry to evaluate hydroxychloroquine and chloroquine retinal toxicity. Can J Ophthalmol 2013; 48: 400-405.

88 Molina-Martin A, Pinero DP, Perez-Cambrodi RJ. Decreased perifoveal sensitivity detected by microperimetry in patients using hydroxychloroquine 
and without visual field and fundoscopic anomalies. J Ophthalmol 2015; 2015: 437271.

89 Jivrajka RV, Genead MA, McAnany JJ, Chow CC, Mieler WF. Microperimetric sensitivity in patients on hydroxychloroquine (Plaquenil) therapy. Eye (Lond) 2013; 27: 1044-1052.

90 Easterbrook M. RE. Microperimetry and the diagnosis of antimalarial maculopathy. Can J Ophthalmol 2014; 49: 307-308.

91 Latasiewicz M, Gourier G, Yusuf IH, Luqmani R, Sharma SM, Downes SM. Hydroxychloroquine retinopathy: an emerging problem. Eye (lond) 2017; 31: 972-976.

92 Akhavan PS, Su J, Lou W, Gladman DD, Urowitz MB, Fortin PR. The early protective effect of hydroxychloroquine on the risk of cumulative damage in patients with systemic lupus erythematosus. J Rheumatol 2013; 40: 831-841.

93 Clowse ME, Magder L, Witter F, Petri M. Hydroxychloroquine in lupus pregnancy. Arthritis Rheum 2006; 54: 3640-3647.

94 Fessler BJ, Alarcon GS, McGwin Jr G, Roseman J, Bastian HM, Friedman AW et al. Systemic lupus erythematosus in three ethnic groups: XVI. Association of hydroxychloroquine use with reduced risk of damage accrual. Arthritis Rheum 2005; 52: 1473-1480.

95 Pons-Estel GJ, Alarcon GS, Gonzalez LA, Zhang J, Vila LM, Reveille JD et al. Possible protective effect of hydroxychloroquine on delaying the occurrence of integument damage in lupus: LXXI, data from a multiethnic cohort. Arthritis Care Res (Hoboken) 2010; 62: 393-400.

96 Schmajuk G, Yazdany J, Trupin L, Yelin E. Hydroxychloroquine treatment in a community-based cohort of patients with systemic lupus erythematosus. Arthritis Care Res (Hoboken) 2010; 62: 386-392.

97 Tang C, Godfrey T, Stawell R, Nikpour M. Hydroxychloroquine in lupus: emerging evidence supporting multiple beneficial effects. Intern Med J 2012; 42: 968-978.
98 Kim HA, Choi HJ, Baek HJ, Lim MJ, Park W, Lee J et al. Nonsteroidal antiinflammatory drugs (NSAID) versus NSAID with hydroxychloroquine in treatment of chemotherapy-related arthropathy: open-label multicenter pilot study. J Rheumatol 2012; 39: 1902-1903.

99 Marchetti M, Baker MG, Noland MM. Treatment of subcutaneous sarcoidosis with hydroxychloroquine: report of 2 cases. Dermatol Online J 2014; 20: 21250.

100 Albers SE, Glass LF, Fenske NA. Lichen planus subtropicus: direct immunofluorescence findings and therapeutic response to hydroxychloroquine. Int J Dermatol 1994; 33: 645-647.

101 Stoll DM. Treatment of cutaneous pseudolymphoma with hydroxychloroquine. J Am Acad Dermatol 1983; 8: 696-699.

102 Khoury H, Trinkaus K, Zhang MJ, Adkins D, Brown R, Vij $\mathrm{R}$ et al. Hydroxychloroquine for the prevention of acute graft-versus-host disease after unrelated donor transplantation. Biol Blood Marrow Transplant 2003; 9: 714-721.

103 Lagneaux L, Delforge A, Carlier S, Massy M, Bernier M, Bron D. Early induction of apoptosis in B-chronic lymphocytic leukaemia cells by hydroxychloroquine: activation of caspase- 3 and no protection by survival factors. Br J Haematol 2001; 112: 344-352.

104 Lagneaux L, Delforge A, Dejeneffe M, Massy M, Bernier M, Bron D. Hydroxychloroquine-induced apoptosis of chronic lymphocytic leukemia involves activation of caspase- 3 and modulation of Bcl-2/bax/ratio. Leuk Lymphoma 2002; 43: 1087-1095.

105 Rosenfeld MR, Ye X, Supko JG, Desideri S, Grossman SA, Brem $S$ et al. A phase I/II trial of hydroxychloroquine in conjunction with radiation therapy and concurrent and adjuvant temozolomide in patients with newly diagnosed glioblastoma multiforme. Autophagy 2014; 10: 1359-1368.

106 Wolpin BM, Rubinson DA, Wang X, Chan JA, Cleary JM, Enzinger PC et al. Phase II and pharmacodynamic study of autophagy inhibition using hydroxychloroquine in patients with metastatic pancreatic adenocarcinoma. Oncologist 2014; 19: 637-638. 